ing courses lasting 6 or 8 weeks. Letters from leading mathematicians to The Times have welcomed some of Prof. Thwaites's proposals, have been severely critical of others, and have suggested alternatives; but none has impugned his statement of the magnitude and urgency of this problem. There is fairly general agreement that an effort must be made to force the country to understand that a shortage of mathematics teachers is not a small, sectional matter, but the symptom of a coming national catastrophe, that, in fact, we may already have passed the point of no return. Although various bodies have seen the danger and have issued warnings, none of them has so far been able to command the attention of the nation as a whole.

If the danger is great-and no one has yet suggested that it is not-then the responsibility for meeting it lies not only on the teaching and research associations, but also on the University Grants Committee, the Ministry of Education and the Ministry for Science. Action at Cabinet-level may be needed ; but a factfinding committee would be useless. There is no time for a minute inquiry into minor statistical detail, and no salvation in a report that the problem is insoluble and so may safely be left to solve itself. A small strong committee, taking for its motto Florence Nightingale's words, "Reports are not self-executive", might yet retrieve the situation.

\section{WAVE PROPAGATION AND GROUP VELOCITY}

\section{Wave Propagation and Group Velocity}

By Léon Brillouin. (Pure and Applied Physics: a Series of Monographs, Vol. 8.) Pp. xi 154. (New York: Academic Press, Inc.; London : Academic Press, Inc. (London), Ltd., 1960.) 6 dollars.

T is difficult to see what purpose this book is meant to serve. Ostensibly it is to make available certain original papers which are not easily accessible because they were published in Germany in 1914 . In his foreword, Dr. Brillouin says : "All these modern developments made it advisable to assemble here a systematic presentation of the original papers, which are rather difficult to find nowadays. It is hoped that the present book will be helpful to many readers and save them time and trouble, especially the trouble of recomputing and rediscovering many important features of the general theory". And again in the preface: "I discussed the solution in great detail and gave a complete answer. . . These problems have come again into the foreground in connection with radio signals and radar. Reflection in the Heaviside layer requires a real knowledge of all these definitions (of group velocity)". But the "modern developments" referred to are in fact very old history now. Of more than sixty references given to the literature only seven are later than 1930, and of these only three are really relevant. The latest reference to propagation in the Heaviside layer is 1931 .

One gets the impression that in Dr. Brillouin's view "les jeunes" have much to learn and little to teach concerning the propagation of waves, and that if they will just take the trouble to read this little book they will find all that is important about the subject. This cannot be accepted. The papers are badly written, and they do not by any means give the "complete answer" even to the limited problem with which they deal.

This is not to say that the book lacks interest. The subject is in fact a fascinating one. When a nearly monochromatic light wave with a sharp front falls on a transparent medium the disturbance that travels through the medium consists of three parts : a first forerunner, travelling with the same speed as light in vacuo; a second forerunner; and then the main signal, travelling with a velocity which is, except near regions of anomalous dispersion, the group velocity.

The forerunners have a very small amplitude in comparison with the main signal. The first forerunner has a very high frequency. If the incident signal rises from zero to a steady sine wave suddenly, then the frequency of the first forerunner is at first much higher than the frequency of the light, and then gets progressively less, the amplitude meanwhile increasing. But if the incident signal is not so idealized, this rapidly oscillating aspect of the first forerunner is blurred. The second forerunners are of lower frequency, comparable with the signal frequency and their onset travels with a velocity characteristic of the medium (and less than the velocity of light in vacuo). They then increase rapidly in amplitude as the main signal arrives, and merge with it.

These deductions from the electromagnetic theory of matter show that no signal travels faster than vacuum light velocity. The first forerunners travel with exactly that velocity. There is, of course, no mystery here. The medium is supposed to consist of electrical particles which on average are at rest before the signal arrives. When it comes, the particles take a little while to get moving, and so to modify the primary field, which, of course, is travelling with vacuum light velocity in the empty space between the electrical constituents of matter.

The book looks at this problem from several slightly different points of view, bringing forward some interesting considerations, in particular the idea of velocity of energy propagation, as distinct from signal velocity, and group velocity. These three velocities are for all practical purposes the same except in regions of anomalous dispersion, where strong absorption of the signal confuses the picture. Unfortunately, much of the value of this discussion is marred by the repetitive nature of the chapters. Had the subject been treated in a unified manner, instead of a 're-hash' of several published papers, the book could have been shorter and very much clearer. There is a deal of inelegant mathematical detail, particularly in Chapter 3, which one presumes to have been Dr. Brillouin's doctor's thesis under Sommerfeld. By contrast, Chapter 2, which is a survey of the subject by Sommerfeld himself, is a masterpiece of clarity and style. It is a pity that Dr. Brillouin did not rewrite the rest of the book nearer to this model. The final chapter is an odd one, having really very little to do with the rest of the book. It deals in a rather cursory manner with wave-guide propagation and looks strangely out of place.

The translation into English from the German and French of the originals is inept. The figures are difficult to follow because the labelling of different curves is not clearly distinguished in the legends. There are many misprints.
M. H. L. PRYor 